\title{
Evaluation du comportement trophique de Aedes aegypti dans la ville de cotonou au sud du Bénin
}

\section{Yadouleton Anges \\ Dramane Gado}

Ecole Normale Supérieure de Natitingou; Université Nationale des Sciences,

Technologies, Ingénierie et Mathématiques (UNSTIM) d'Abomey

\section{Ahadji-Dabla K.M.}

Département de Zoologie et de Biologie Animale. Faculté des Sciences, Université de Lomé. Biodiversity Institute and Department of Ecology and Evolutionary Biology, University of Kansas, USA

\section{Agbanrin Ramziyath \\ Tchibozo Carine \\ Agolinou Achaz \\ Tossou Roland}

Centre de Recherches Entomologiques de Cotonou, Cotonou, Benin Baba-Moussa Lamine

Laboratoire de Biologie et de Typage Moléculaire en Microbiologie, Faculté des Sciences et Techniques, Université d'Abomey-Calavi

Doi: 10.19044/esj.2018.v14n33p70 URL:http://dx.doi.org/10.19044/esj.2018.v14n33p70

\begin{abstract}
In order to evaluate the trophic behavior of Aedes aegypti in the city of Cotonou, southern Benin, a cross-sectional study was conducted in urban and periurban neighborhoods from April 2016 to August 2017 to collect Ae. Aegypti populations. To achieve this goal, day and night collections, twice a month for one year were done where Ae. Aegypti populations were caught by Human Landing Catch and BG-Sentinel traps. 3,892 Aedes caught by different methods showed that Ae. Aegypti and Ae. circumluteolus are more abundant in urban than peri-urban areas $(\mathrm{p}<0.05)$. The aggressive density of Ae. aegypti populations was significantly higher in urban areas (157.43 bites per man per hour) than in peri-urban areas (32.43 bites per man per hour) ( $\mathrm{p}<0.05)$. Also, out of 250 females blood-fed Ae. Aegypti tested by ELISA (Enzyme Linked Immunosorbent Assay) technique for blood meal identification, $86.80 \%$ took their blood meal on humans compared to $4.4 \%$ which took their blood meal on sheeps. These findings showed that the city of Cotonou in southern Benin, offered good condition for the development of
\end{abstract}


Ae. aegypti population. The anthropology and endophagy behavior of this mosquito observed through the results of this study is a very favorable clue to vector control strategies based on the use of long-lasting impregnated mosquito nets and insecticide residual sprays adopted in Benin.

Keywords: Aedes aegypti, collection, behavior, Cotonou, Benin

\section{Résumé}

Dans le but d'évaluer le comportement trophique de Aedes aegypti dans la ville de Cotonou au sud du Bénin, une étude a été conduite dans les quartiers urbains et periurbains d'Avril 2016 à Mars 2017 afin de capturer les populations de Ae. Aegypti. Pour atteindre cet objectif, des captures sur appât humain et à partir de pièges BG-Sentinel ont été faites de jour comme de nuit deux fois par mois et ceci pendant une année. Il ressort des collectes que sur un total de 3.892 Aedes spp capturés, Ae. aegypti et Ae. circumluteolus ont été plus abondantes dans les quartiers urbains que périurbains $(\mathrm{p}<0,05)$. La densité agressive des populations de Ae. aegypti a été significativement plus importante dans la zone urbaine (157,43 piqûres par homme par heure) qu'en zone périurbaine $(32,43$ piqûres par homme par heure) $(\mathrm{p}<0,05)$. Aussi, sur 250 femelles de Ae. Aegypti gorgées et testées à l'ELISA (Enzyme Linked Immunosorbent Assay), 86,80\% ont pris leur repas sanguin chez 1'homme contre $4,4 \%$ qui ont pris leur repas sur les moutons. Ces résultats montrent que la situation écologique de la ville de Cotonou est favorable au maintien d'une densité importante de la population de Ae. Aegypti et dont l'appât préférentiel reste l'homme. Le caractère anthropo-endophage de ce moustique constaté à travers les résultats de cette étude constitue un indice très favorable aux stratégies de lutte anti-vectorielle basée sur l'utilisation des Moustiquaires Imprégnées à Longue Durée d'Action et les pulvérisations intra-domiciliaires adoptées depuis quelques années au Bénin.

Mots clés: Aedes aegypti, comportement, capture ; Cotonou, Bénin

\section{Introduction}

Plusieurs maladies à transmission vectorielle sont causées par les arthropodes et, les plus importantes se retrouvent en Afrique subsaharienne (WHO, 2016). Parmi les vecteurs de ces maladies, les moustiques sont les plus redoutables, tant par leur abondance que par les maladies qu'ils transmettent (WHO, 2016). Les principaux genres de moustiques qui sont à la base de ces pathologies sont : Anopheles, Aedes et Culex.

Le Bénin, comme l'ensemble des pays de l'Afrique subsaharienne, paie un lourd tribut aux maladies à transmission vectorielle (Akogbeto et al., 
2010). En effet, la ville de Cotonou à l'image des grandes villes d'Afrique a connu des modifications profondes tant sur le plan démographique qu'infrastructurelle, marquée par une urbanisation galopante. Cette urbanisation galopante et une pluviométrie importante ont favorisé l'émergence de plusieurs maladies vectorielles dans plusieurs villes du Bénin (Yadouleton et al., 2014). Ces différents facteurs exposent Cotonou à de grandes épidémies urbaines comme la fièvre de la dengue transmise par Aedes aegypti (Yadouleton et al., 2014). Ce moustique domestique très hématophage se trouve dans les habitations humaines et, est présent dans les jarres, boîtes de conserves abandonnées, du nord au sud du Bénin durant toute l'année avec des risques de propagation de la fièvre de la dengue (Yadouleton et al., 2018). C'est une maladie virale causée par un virus de la famille des flaviviridae appartenant à quatre stéréotypes (DEN-1 à - 4) et transmise par la piqûre diurne de moustique du genre Aedes spp. Son extension récente et rapide à de nouvelles zones géographiques, y compris aux milieux ruraux fait qu'elle est considérée comme un problème de santé publique (WHO, 2016). En effet, au cours des cinq dernières années, plusieurs cas de recrudescences de cette fièvre ont été signalés dans plusieurs pays de l'Afrique de l'Ouest notamment au Burkina en 2016 et 2017 ( 2680 cas suspects et 20 cas de décès enregistrés). Outre ce pays, la Côte d'Ivoire a été aussi touchée en 2017 avec 911 cas suspects notifiés dans les établissements sanitaires d'Abidjan où 311 cas de dengue ont été confirmés (WHO, 2017).

Les symptômes de la maladie, similaire à ceux du paludisme, sont souvent sous diagnostiqués au Bénin du fait de l'inexistence de laboratoire adéquat et de personnels qualifiés. Or, les travaux de Mugen Ujiie et al. (2012) et Moi et al. (2010) ont montré la présence de la fièvre de la dengue de stéréotypes 2 e t 3 a u p è s des touristes japonais et français ayant séjourné au Bénin. Dès lors, la lutte contre ce moustique s'avère indispensable. C'est dans ce cadre que la présente étude a été initiée afin de disposer de données liées à l'évaluation du comportement trophique couplée à la densité agressive et au type de repas sanguin de Aedes aegypti, et mieux cerner ce moustique dans son biotope pour une bonne lutte.

\section{Matériel et méthodes}

\section{Zone d'étude}

La présente étude a été réalisée dans la ville de Cotonou $\left(6^{\circ} 20^{\prime}\right.$ et $6^{\circ} 23^{\prime}$ de latitude Nord et $2^{\circ} 22^{\prime}$ et $2^{\circ} 30^{\prime}$ de longitude Est) et plus précisément dans les quartiers urbains et périurbains de cette ville située sur le cordon littoral qui s'étend entre le lac Nokoué et l'Océan atlantique (Figure 1). La ville a un 
climat subéquatorial où la température moyenne est de $27,3^{\circ} \mathrm{C}$ avec une pluviométrie relativement abondante (1300 $\mathrm{mm}$ par an en moyenne). Les précipitations ont un régime bimodal et ont lieu principalement entre mars et juillet et atteignent leur maximum en juin.

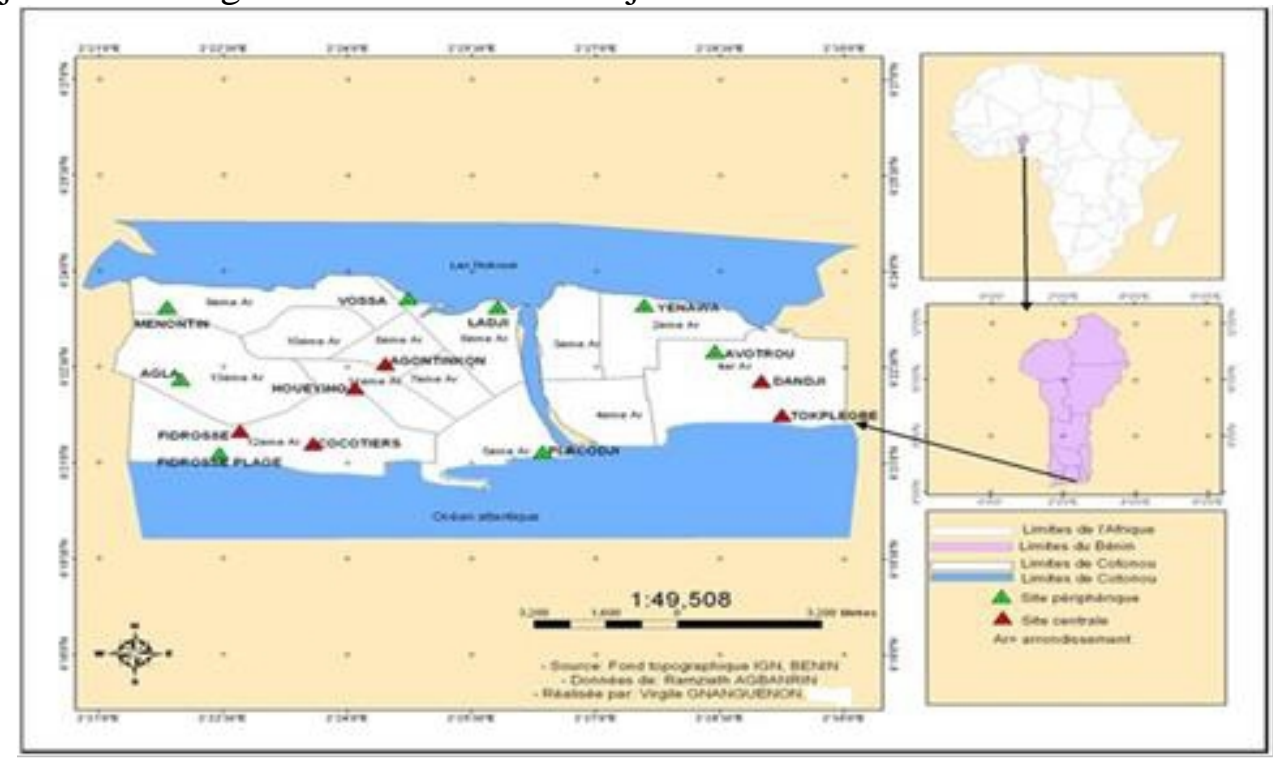

Figure 1 : Carte du Bénin indiquant le site d'étude.

\section{Collecte des moustiques}

L'étude du comportement trophique de Aedes aegypti a été réalisée à partir des captures mensuelles de moustiques d'Avril 2016 à Mars 2017 dans les habitations humaines habitées. Ces captures ont été réalisées deux fois par mois, le jour et la nuit, de façon consécutive.

Les captures du jour se sont déroulées de $14 \mathrm{~h}$ à $18 \mathrm{~h}$ et celles de nuit de $20 \mathrm{~h}$ à $5 \mathrm{~h}$. Deux équipes de quatre captureurs chacune ont travaillé à l'intérieur et à l'extérieur de quatre habitations dans chaque quartier des zones urbaines et périurbaines. Une équipe pour les captures du jour et l'autre pour les captures de nuit. Les captureurs ont subi une rotation dans les différentes habitations pour éviter les biais liés à leur habileté ou à leur attractivité individuelle. Tous les captureurs ont été vaccinés contre la fièvre jaune et protégés du paludisme par une chimioprophylaxie à la sulfadoxine pyriméthamine. Par ailleurs, deux maisons ont été choisies dans chaque quartier le lendemain pour la récolte de la faune résiduelle entre $06 \mathrm{~h}$ et $07 \mathrm{~h}$, à la suite de la pulvérisation intradomiciliaire de pyréthrine après chaque séance de capture de nuit. Dans deux autres maisons de chacun des quartiers, deux pièges BG-Sentinel, à raison d'un piège par maison, ont été posés à l'extérieur des chambres pour la capture des moustiques de $18 \mathrm{~h}$ à $07 \mathrm{~h}$ du matin. Tous les moustiques capturés ont été identifiés morphologiquement à l'aide des clés 
d'identification de Edwards (1941) et de Gillies et de Meillon (1968), puis conservés dans des tubes eppendorff sur du silicagel à $-20^{\circ} \mathrm{C}$ jusqu'à leur traitement. L'identification de l'origine des repas de sang pris par les femelles de Aedes aegypti a été effectuée par la technique ELISA directe (EnzymeLinked Immunosorbent Assay) décrite par Beier et al (1998).

\section{Analyse statistique}

L'abondance relative $(\mathrm{p} i=\mathrm{ni} / \mathrm{N}$ où ni=effectif de l'espèce de rang $\mathrm{i}$, $\mathrm{N}=$ effectif total) de chaque espèce a été exprimée telle que définie par Barbault (1992). L'analyse des données a été réalisée avec le logiciel R-2.15.2 (DICICCIO T.J., EFRON B. (1996).

La densité agressive [nombre de piqûre/homme/nuit] (ma) à l'intérieur et à l'extérieur des habitations, le taux d'endophagie, et le taux de gorgement des différentes espèces durant les saisons sèche et pluvieuse dans les zones urbaines et périurbaines ont été déterminés. Les comparaisons de ces taux ont été faites par le test de $\mathrm{Chi}^{2}$.

\section{Résultats}

\section{Abondance des espèces de moustiques collectés}

Un total de 45.609 moustiques a été capturé par différentes méthodes. De façon générale, Culex quinquefasciatus Say, Aedes aegypti, et Anopheles gambiae s.l ont été les espèces dominantes dans les deux zones urbaines et périurbaines de la ville avec des abondances relatives qui sont respectivement de 64,$30 ; 22,86$ et $12,84 \%$ (Tableau 1). La population de $C x$. quinquefasciatus a été plus abondante dans la zone périurbaine que dans la zone urbaine avec respectivement 76,27\% et 49,24\% $\quad(\mathrm{p}<0,05)$ (Tableau 1). Les espèces $A e$. luteocephalus, Ae. vitattus, An. gambiae s.l, An. ziemanni, An . pharoensis, Cx. tigripes, $C x$. fatigrans, $C x$. nebulosus et Mansonia africana ont été aussi plus abondantes dans la zone périurbaine que urbaine. $(\mathrm{p}<0,05)$. Par contre, Ae. Aegypti et Ae. circumluteolus ont été plus abondantes dans les quartiers urbains que périurbains $(\mathrm{p}<0,05)$. Les espèces $C x$. decens et Uranotaenia bilineata n'ont pas montré de variation significative de leurs abondances relatives entre les zones urbaine et périurbaine $(\mathrm{p}>0,05)$. Signalons que $M$. uniformis a été absent en milieu urbain et faiblement représenté en milieu périurbain avec une abondance relative de 0,01\% ( $>>0,05)$. De façon globale, le total des moustiques capturés est plus important en zone périurbaine (25.303) qu'en zone urbaine (20.306) $(\mathrm{p}<0,0001)$ (tableau 1). 
Tableau 1 : Abondance des espèces de moustiques dans les zones urbaines et périurbaines de Cotonou

\begin{tabular}{|c|c|c|c|c|c|c|c|c|}
\hline \multirow{2}{*}{ Genres } & \multirow{2}{*}{ Espèces } & \multicolumn{2}{|c|}{ Zone périurbaine } & \multicolumn{2}{|c|}{ Zone urbaine } & \multicolumn{2}{|l|}{ Total } & \multirow{2}{*}{$\mathrm{P}$} \\
\hline & & $\mathrm{ABR}$ & $\operatorname{ABR}(\%)$ & $\mathrm{ABR}$ & $\operatorname{ABR}(\%)$ & $\mathrm{ABR}$ & $\operatorname{ABR}(\%)$ & \\
\hline \multirow{4}{*}{ Aedes } & luteocephalus & 79 & 0,33 & 22 & 0,11 & 101 & 0,23 & 0,0004 \\
\hline & aegypti & 2187 & 9 & 7781 & 40,3 & 9968 & 22,86 & $<0,0001$ \\
\hline & circumluteolus & 41 & 0,17 & 61 & 0,32 & 102 & 0,23 & 0,002 \\
\hline & vittatus & 10 & 0,04 & 0 & 0 & 10 & 0,02 & 0,0108 \\
\hline \multirow{3}{*}{ Anopheles } & gambiae s.l & 2839 & 14,56 & 2285 & 11,93 & 5124 & 12,84 & $<0,0001$ \\
\hline & pharoensis & 163 & 0,67 & 98 & 0,51 & 261 & 0,6 & 0,03312 \\
\hline & ziemanni & 96 & 0,4 & 29 & 0,15 & 125 & 0,29 & $<0,0001$ \\
\hline \multirow{5}{*}{ Culex } & tigripes & 319 & 1,31 & 146 & 0,76 & 465 & 1,07 & $<0,0001$ \\
\hline & fatigans & 77 & 0,32 & 23 & 0,12 & 100 & 0,23 & $<0,0001$ \\
\hline & gr. decens & 33 & 0,14 & 25 & 0,13 & 58 & 0,13 & 0,9627 \\
\hline & nebulosus & 264 & 1,09 & 167 & 0,87 & 431 & 0,99 & 0,02312 \\
\hline & quinquefasciatus & 18535 & 76,27 & 9507 & 49,24 & 28042 & 64,3 & $<0,0001$ \\
\hline \multirow{2}{*}{ Mansonia } & africana & 544 & 2,24 & 214 & 1,11 & 758 & 1,74 & $<0,0001$ \\
\hline & uniformis & 3 & 0,01 & 0 & 0 & 3 & 0,01 & 0,2573 \\
\hline \multirow[t]{2}{*}{ Uranotaenia } & bilineata & 114 & 0,47 & 89 & 0,46 & 203 & 0,47 & 0,9778 \\
\hline & Abondance & 25303 & 100 & 20306 & 100 & 45609 & 100 & $<0,0001$ \\
\hline
\end{tabular}

$\mathrm{ABR}=$ Abondance, $\mathrm{ABR}(\%)=$ Abondance relative

\section{Densités agressives moyennes des femelles de Aedes aegypti Capture diurne}

Un total de 3.892 Aedes aegypti a été collecté sur homme en 16 séances de capture dirurne. La densité agressive des populations de Ae. aegypti a été significativement plus importante dans la zone urbaine (157,43 piqûres par homme par heure) qu'en zone périurbaine $(32,43$ piqûres par homme par heure) en saison pluvieuse $(\mathrm{p}<0,05)$ (Tableau 2).

Tableau 2 : Densités agressives moyennes des femelles de Aedes aegypti dans les zones urbaine périurbaines en fonction des saisons à l'issue de la capture diurne.

\begin{tabular}{|c|c|c|c|c|c|c|c|c|c|c|}
\hline & & & \multicolumn{4}{|c|}{ Saison Sèche } & \multicolumn{4}{|c|}{ Saison Pluvieuse } \\
\hline Zones & Espèces & & $\begin{array}{l}\text { Nombre } \\
\text { de } \\
\text { vecteurs }\end{array}$ & $\begin{array}{l}\text { Nombre } \\
\text { de } \\
\text { captureurs }\end{array}$ & $\begin{array}{l}\text { Piqûre / } \\
\text { homme/ } \\
\text { Heure }\end{array}$ & $\mathbf{P}$ & $\begin{array}{l}\text { Nombre } \\
\text { de } \\
\text { vecteurs }\end{array}$ & $\begin{array}{l}\text { Nombre } \\
\text { de } \\
\text { captureurs }\end{array}$ & $\begin{array}{l}\text { Piqûre/ } \\
\text { homme/ } \\
\text { heure }\end{array}$ & $\mathbf{P}$ \\
\hline \multirow{2}{*}{ urbaine } & & Ext & 198 & 16 & 12,38 & \multirow{2}{*}{$<0.001$} & 328 & 16 & 20,5 & \multirow{2}{*}{$<0.001$} \\
\hline & $a e$ & Int & 496 & 16 & 31 & & 2198 & 16 & 137,38 & \\
\hline \multirow{2}{*}{ Périurbaine } & Aedes & Ext & 57 & 16 & 3,57 & \multirow{2}{*}{0.002} & 120 & 16 & 7,5 & \\
\hline & aegypti & Int & 89 & 16 & 5,57 & & 406 & 16 & 25,38 & $<0.001$ \\
\hline
\end{tabular}

\section{Capture nocturne}

Les résultats du Tableau 3 montrent que la densité agressive de Ae. Aegypti est significativement plus importante en saison pluvieuse (622 piqûres 
par homme et par nuit) qu'en saison sèche (334 piqûres par homme et par nuit) $(\mathrm{P}<0,05)$.

Tableau 3 : Densités agressives moyennes des femelles de Aedes aegypti dans les zones urbaine et périurbaine en fonction des saisons à l'issue de la capture nocturne.

\begin{tabular}{|c|c|c|c|c|c|c|c|c|c|c|}
\hline \multirow[b]{2}{*}{ Zones } & \multirow[b]{2}{*}{ Espèces } & & \multicolumn{4}{|c|}{ Saison Sèche } & \multicolumn{4}{|c|}{ Saison Pluvieuse } \\
\hline & & & $\begin{array}{l}\text { Nombre } \\
\text { de } \\
\text { vecteurs }\end{array}$ & $\begin{array}{l}\begin{array}{l}\text { Nombre } \\
\text { de }\end{array} \\
\text { captureurs }\end{array}$ & $\begin{array}{l}\text { Piqûre / } \\
\text { homme/ } \\
\text { Nuit }\end{array}$ & $\mathbf{P}$ & $\begin{array}{l}\text { Nombre } \\
\text { de } \\
\text { vecteurs }\end{array}$ & $\begin{array}{l}\text { Nombre } \\
\text { de } \\
\text { captureurs }\end{array}$ & $\begin{array}{l}\text { Piqûre/ } \\
\text { homme/ } \\
\text { nuit }\end{array}$ & $\mathbf{P}$ \\
\hline \multirow{2}{*}{ Urbaine } & Aedes & Ext & 185 & 16 & 11,57 & \multirow{2}{*}{$<0.001$} & 228 & 16 & 14,25 & \multirow{2}{*}{$<0.001$} \\
\hline & aegypti & Int & 86 & 16 & 5,38 & & 198 & 16 & 12,38 & \\
\hline \multirow{2}{*}{ Périubaine } & Aedes & Ext & 38 & 16 & 2,38 & \multirow{2}{*}{0.0029} & 120 & 16 & 7,5 & \multirow{2}{*}{$<0.001$} \\
\hline & aegypti & Int & 25 & 16 & 1,57 & & 76 & 16 & 4,75 & \\
\hline
\end{tabular}

Fluctuations saisonnières du taux d'endophagie des femelles de Aedes aegypti

Pour un total de 4.650 femelles de Ae. aegypti, le taux d'endophagie a été de 76,86\% au cours des saisons. En effet, 696 moustiques sur 1174 ont pris leur repas de sang à l'intérieur des habitations pendant la saison sèche et 2878 sur 3476 au cours de la saison pluvieuse (tableau 4). Le taux d'endophagie de Ae. aegypti a été significativement plus élevé au cours de la saison pluvieuse $(87 \%)$ qu'en saison sèche $(60,31 \%)$ dans la zone urbaine $(\mathrm{p}<0.0001)$. Le même constat a été fait en zone périurbaine.

Tableau 4: Variation saisonnière du taux d'endophagie des femelles de Aedes aegypti en zones urbaines et périurbaines de la ville de Cotonou

\begin{tabular}{|c|c|c|c|c|c|c|c|c|c|c|}
\hline \multirow[b]{2}{*}{ Espèces } & \multicolumn{4}{|c|}{ Saison Sèche } & \multicolumn{6}{|c|}{ Saison Pluvieuse } \\
\hline & Zones & $\begin{array}{l}\mathbf{N} \\
\text { Vect* }\end{array}$ & Total & $\begin{array}{l}\text { Endo } \\
(\%)\end{array}$ & $\mathbf{P}$ & $\mathbf{P}^{\mathrm{S}}$ & $\begin{array}{l}\mathbf{N} \\
\text { Vect* }\end{array}$ & Total & $\begin{array}{l}\text { Endo } \\
(\%)\end{array}$ & $\mathbf{P}$ \\
\hline Ae aeovnti & & 114 & 209 & 54,55 & $<00001$ & $<0,0001$ & 482 & 722 & 66,75 & $<00001$ \\
\hline Total & urbaine & $\begin{array}{l}582 \\
696\end{array}$ & $\begin{array}{l}965 \\
1174\end{array}$ & $\begin{array}{l}60,31 \\
59,28\end{array}$ & & $<0,0001$ & $\begin{array}{l}2396 \\
2878\end{array}$ & $\begin{array}{l}2754 \\
3476\end{array}$ & $\begin{array}{l}87 \\
82,80\end{array}$ & \\
\hline
\end{tabular}

* : Nombre de moustiques collecté à l'intérieur des maisons. Endo (\%) : taux endophagie ; P : p-value des zones ; PS : p-value des saisons

Variation saisonnière du taux de gorgement de Aedes aegypti

Les résultats obtenus ont montré que pendant la saison sèche, il n'existe pas de différence significative entre le taux de gorgement de $A e$. aegypti en zone urbaine et périurbaine ( $\mathrm{p}>0,05)$. (Tableau 5). Par contre en saison pluvieuse, le taux de gorgement dans la zone urbaine $(56,71 \%)$ est significativement plus important que celui obtenu en zone périurbaine $(48,91 \%)(\mathrm{p}<0,0001)$. (Tableau 5). 
Tableau 5 : Variation saisonnière du taux de gorgement des femelles de Aedes aegypti dans les zones urbaine et périurbaine de Cotonou

\begin{tabular}{lllllccccccc}
\hline & \multicolumn{4}{c}{ Saison Sèche } & \multicolumn{1}{c}{ Saison Pluvieuse } \\
\hline \multirow{2}{*}{ Espèces } & Zones & N & Gorg & Total & Gorg(\%) & P & Ps & Gorg & Total & Gorg(\%) & P \\
\hline \multirow{2}{*}{ Ae. aegypti } & périurbaine & 63 & 159 & 39,62 & \multirow{2}{*}{0.2123} & 0,14 & 67 & 137 & 48,91 & $<0,0001$ \\
& urbaine & 58 & 176 & 32,95 & & $<0,0001$ & 77 & 136 & 56,71 & & \\
\hline
\end{tabular}

$\mathrm{N}$ Gorg : nombre de moustiques gorgés ; $\operatorname{Gorg}(\%)$ : taux gorgement $\mathrm{P}: \mathrm{p}$-value des zones ; $\mathrm{P}^{\mathrm{S}}$ : p-value des saisons

\section{Préférence trophique des femelles de Aedes aegypti}

Un total de 250 femelles de Ae. Aegypti gorgées a été testé à l'ELISA (tableau 6). Les résultats obtenus ont montré que $86,80 \%$ des populations de Ae.aegypti ont pris leur repas sanguin sur l'homme contre $4,4 \%$ qui ont pris leur repas sur les moutons.

Tableau 6 : Origine du repas de sang des femelles de Aedes aegypti de capturés à l'intérieur des maisons dans la ville de Cotonou

\begin{tabular}{|c|c|c|c|c|c|c|c|}
\hline \multirow[t]{2}{*}{ Espèc } & \multirow[t]{2}{*}{ Identifiés } & Homme & Bœuf & Mouton & Porc & \multirow{2}{*}{ Poule } & \multirow{2}{*}{$\begin{array}{l}\text { Négatifs } \\
\mathrm{n} \%\end{array}$} \\
\hline & & $\%$ & $\mathrm{n} \quad \%$ & $\%$ & $\mathrm{n} \quad \%$ & & \\
\hline
\end{tabular}

\begin{tabular}{llllllllllllll} 
Ae. aegypti & 250 & 217 & $86,80^{\mathrm{a}}$ & 0 & $0^{\mathrm{b}}$ & 11 & $4,4^{\mathrm{c}}$ & 0 & $0^{\mathrm{b}}$ & 0 & $0^{\mathrm{b}}$ & 22 & 8,8 \\
\hline
\end{tabular}
Les pourcentages affectés d'une même lettre ne sont pas significativement différents $(p>0,05)$. Ceux affectés des lettres différentes sont différents significativement $(p<0,05)$.

\section{Discussion}

L'étude du comportement trophique de Aedes aegpti est indispensable dans la mise en place de stratégies pour lutter contre ce moustique dans la ville de Cotonou. La présente étude conduite sur douze mois dans la ville de Cotonou a montré que Aedes aegpti est présent au cours des deux saisons dans les zones urbaines et périurbaines et confirme les résultats des travaux de Yadouleton et al (2014). Cette présence pourrait s'expliquer par la mauvaise urbanisation créant de nombreux gîtes domestiques favorables au le développement de Ae. Aegypti. A cela il faut ajouter les gîtes péri domestiques constitués le plus souvent par des pots de fleurs dans les maisons, des citernes dans les chantiers en constructions, des pneus usagés et qui constituent aussi des endroits favorables non seulement à la ponte des œufs de ce moustique mais aussi pour son développement (Yadouleton et al., 2014).

Par ailleurs, si la population de Ae. Aegypi collectée est plus importante pendant le jour que la nuit, nos travaux de recherche à l'instar de ceux de Harrington et al. (2001), confirment l'activité diurne de ce moustique avec une activité plus importante en zone urbaine qu'en zone périurbaine. La manière la plus plausible d'expliquer la forte activité de Ae. Aegypti en zone urbaine 
est d'admettre l'existence de plusieurs appâts en zone urbaine. En effet, l'urbanisation galopante dans la ville de Cotonou associée à l'exode rural ont favorisé l'installation de plusieurs appâts comme l'homme, les animaux domestiques (moutons, bœufs, porcs) en zone urbaine dans des conditions insalubres mais favorables à Ae. Aegypti (Yadouleton et al., 2018 ; Lingenfelser et al., 2010).

L'évaluation des préférences trophiques de Ae. Aegypti effectuée dans cette étude montre que ce moustique est plus anthropophage que zoophage. Ce résultat corrobore ceux de Tradieux et al. (1990) qui avaient montré que Ae. aegypti s'est nourrit presqu'exclusivement sur l'homme à 93\%. Le pourcentage faible (4.4\%) de Ae. Aegypti ayant pris le sang animal dans notre site d'étude montre que ce moustique en absence de son appât préféré (homme), devient zoophage. Cependant, les $86 \%$ des populations de $A e$. Aegypti en provenance du site d'étude et ayant pris leur repas sanguin chez l'homme constituent un indice important non seulement dans la lutte contre ce moustique mais aussi les risques d'arbovirose que courent les populations de la ville de Cotonou.

\section{Conclusion}

Dans la ville de Cotonou la situation écologique est favorable au maintien d'une densité importante de la population de Ae. Aegypti dont l'appât préférentiel reste l'homme. Le caractère anthropo-endophage de ce moustique, constaté à travers les résultats de cette étude constitue un indice très favorable aux stratégies de lutte anti-vectorielle basée sur l'utilisation des Moustiquaires Imprégnées à Longue Durée d'Action et les Pulvérisations intra-domiciliaires adoptées depuis quelques années au Bénin pour lutter contre les populations de moustique.

\section{References:}

1. WHO. 2016. World Malaria Report (2016). World Health Organization, Geneva.

2. AKOGBETO M., PADONOU G.G., GBÉNOU D., IRISH S., YADOULETON A. (2010). Bendiocarb, a potential alternative against pyrethroid resistant Anopheles gambiae in Benin, West Africa. Malar J. 9: 204. doi: 10.1186/1475-2875-9-204

3. YADOULETON A., AGBANRIN R., VODOUNON C., PADONOU G., BADIROU K., ATTOLOU R., URSINS F., ZOLA J., ALLAGBÉ H., AKOGBÉTO M. (2014). Seasonal distribution of Aedes aegypti in southern Benin: a risk of dengue virus transmission to urban populations. Int. J. Innov.Appl. Stud. 648-654. 
4. YADOULETON A., TCHIBOZO C., AGOLINOU A., KPODO E., KPODJEDO J., BANKOLE H., GBAGUIDI F. (2018). Dengue fever immunoglobulin $\mathrm{G}$ (IgG) isotype in blood samples from hospital in Benin. World Wide J. Multidisc. Res. Dev. 4(8): 15-18.

5. WHO. (2017). World Malaria Report. World Health Organization, Geneva

6. UJIIE M., MOI, M.L., KOBAYASHI T., TAKESHITA N., KATO Y., TAKASAKI T., KANAGAWA S. (2012). Dengue virus type-3 infection in a traveler returning from Benin to Japan. J. Travel Med., 19 (4), 255-257. doi: 10.2149/tmh.2014-34

7. MOI M.L, TAKASAKI T, KOTAKI A, TAJIMA S, LIM C.K., SAKAMOTO M, IWAGOE H., KOBAYASHI K., KURANE I.. 2010. Importation of dengue virus type 3 to Japan from Tanzania and Côte d'Ivoire. Emerg. Infect. Dis., 16 (11):1770-1772. doi: 10.3201/eid1611.101061.

8. EDWARDS F. 1941. Mosquitoes of the Ethiopian Region III. Culicine adults and pupae. British Museum (Nat Hist), London.

9. GILLIES M.T., DE MEILLON B. (1968). The Anophelinae of Africa South of the Sahara (Ethiopian Zoogeographical Region). Sth. Afr. Inst. Med. Res. 54, 343.

10. BEIER J., PERKKINS P.V., WIRTZ R.A., KOROS J., DIGGS D., GARGAN T.P., KOECH D.K. (1998). Blood- meal identification by direct-enzyme linked immunosorbent assay (ELISA), tested on Anopheles (Diptera: Culicidae) in Kenya. J. Med. Entomol., 25:9-16. DOI: $10.1093 /$ jmedent/25.1.9

11. BARBAULT R. (1992). Écologie des peuplements : structure, dynamique et évolution. Éditions Masson, Paris, France

12. DICICCIO T.J., EFRON B. (1996). Bootstrap confidence intervals (with Discussion). Stat. Sci.., 11, 189-228

13. HARRINGTON L.C., EDMAN J.D., SCOTT T.W. (2001). Why do female Aedes aegypti (Diptera: Culicidae) feed preferentially and frequently on human blood? J. Med. Entomol., 38, 411-422.

14. LINGENFELSER A., RYDZANICZ K., KAISER A., ACHIM KAISER, BECKER N. (2010). Mosquito fauna and perspectives for integrated control of urban vector-mosquito populations in Southern Benin (West Africa).Ann. Agric. Environ. Med. , 17(1): 49-57. PMID: 20684480

15. TARDIEUX I., POUPEL O., LAPCHIN L., RODHAIN F. (1990). Variation among strains of Aedes aegypti in susceptibility to oral infection with dengue virus type 2. Am. J. Trop. Med. Hyg., 43 (3): 308-13. 